\title{
Telemetry Equipment Device
}

National Cancer Institute

\section{Source}

National Cancer Institute. Telemetry Equipment Device. NCI Thesaurus. Code C50211.

Equipment designed to transmit and receive data from a remote source using telecommunications methods. 